\title{
Microfluidic bioreactors for enzymatic synthesis in packed-bed reactors-Multi-step reactions and upscaling
}

\author{
Eduardo J.S. Brás ${ }^{\mathrm{a}, \mathrm{b}, 1}$, Cristiana Domingues ${ }^{\mathrm{a}, \mathrm{b}, 1}$, Virginia Chu ${ }^{\mathrm{a}}$, Pedro Fernandes ${ }^{\mathrm{b}, \mathrm{c}, \mathrm{d}}$, \\ João Pedro Conde ${ }^{\mathrm{a}, \mathrm{c}, *}$
}

${ }^{\text {a }}$ Instituto de Engenharia de Sistemas e Computadores - Microsistemas e Nanotecnologias (INESC MN), Lisbon, Portugal

${ }^{\mathrm{b}}$ IBB - Institute for Bioengineering and Biosciences, Instituto Superior Técnico, Universidade de Lisboa, Lisbon, Portugal

${ }^{\mathrm{c}}$ Department of Bioengineering, Instituto Superior Técnico, Universidade de Lisboa, Lisbon, Portugal

${ }^{\mathrm{d}}$ DREAMS and Faculty of Engineering, Universidade Lusófona de Humanidades e Tecnologias, Lisbon, Portugal

\section{A R T I C L E I N F O}

\section{Keywords:}

Microfluidics

Biocatalysis

L-DOPA

Dopamine

Cascade reactions

Packed-bed reactor

\begin{abstract}
A B S T R A C T
Enzymatic synthesis of biochemical commodities is of upmost importance as it represents a greener alternative to traditional chemical synthesis and provides easier downstream processing strategies compared to fermentationbased processes. A microfluidic system used to optimize the enzymatic production of both levodopa (L-DOPA) and dopamine in both single-step and multistep-reaction sequences with yield of approximately $30 \%$ for LDOPA production and $70 \%$ for dopamine production is presented. The system for L-DOPA production was then up-scaled (780-fold increase) to a milliliter scale system by maintaining similar mass transport properties resulting in the same yield, space-time yield and biocatalyst yield as its microscale counterpart. The results obtained for yield and biocatalyst yield $\left(351.7 \mathrm{mg}_{\mathrm{L}-\mathrm{DOPA}} \mathrm{mg}_{\mathrm{Tyr}}^{-1} \mathrm{~h}^{-1}\right)$ were similar to what is reported in the literature for similar systems, however the space-time yield $\left(0.806 \mathrm{mg}_{\mathrm{L}-\mathrm{DOPA}} \mathrm{L}^{-1} \mathrm{~h}^{-1}\right)$ was smaller. This work demonstrates a microfluidic bioreactor that can be used for complex optimizations that can be performed rapidly while reducing the consumption of reagents by immobilizing the catalyst on a carrier which can then be used in a packed-bed reactor, thus extending the enzyme life span.
\end{abstract}

\section{Introduction}

Most (bio)chemical commodities today are produced using a biological approach, either by fermentation or by enzymatic synthesis. These goods range from pharmaceutical compounds to everyday commodities such as cosmetics, cleaning products and alcoholic beverages (Choi et al., 2015; Liu, 2012; den Haan et al., 2015). Enzymatic synthesis or biocatalysis is widely used for the production of relevant molecules such as steroids (Wang et al., 2019) and other pharmaceutical compounds (Choi et al., 2015; Sun et al., 2018; Fuchs et al., 2015). This leads to a need to reduce the cost of the production of these compounds, as well as, the development of efficient screening systems for process optimization, two areas where microfluidics could play an important role.

Reports of the use of microfluidic structures for the screening of production conditions for both single step enzymatic reactions as well as chemo-enzymatic and enzymatic cascade systems are found in the literature (Gruber et al., 2017; Strniša et al., 2018). Microfluidic platforms were used in the production of neohesperidin ester derivatives using lipase (Du et al., 2018), the chemo-enzymatic synthesis of 2-aminophenoxazin-3-one (Luckarift et al., 2007) and in the production of chiral amino-alcohols through an enzymatic cascade (Gruber et al., 2018). These microfluidic platforms may consist of simple systems where the enzymes are either immobilized on the microfluidic channel wall or in flow as free enzymes (Zhu et al., 2019), or more complex designs involving the use of enzymatic carriers, such as polymeric capsules or glass particles functionalized with the enzyme of interest (Asanomi et al., 2011).

Microreactors can also be used for the actual production instead of just a tool to expedite optimization processes. These systems take advantage of the continuous mode of operation enabled by the microfluidic structures, with some examples being the work of Madarász et al., where they report the production of isoamyl oleate in a continuous enzymatic microreactor (Madarász et al., 2015), and the work of Giovannini et al., for the production of eugenol esters in microfluidic bioreactors (Giovannini et al., 2019), among others (Gruber et al.,

\footnotetext{
* Corresponding author.

E-mail address: joao.conde@tecnico.ulisboa.pt (J.P. Conde).

${ }^{1}$ Authors with equal contribution.
} 
2018; Bolivar et al., 2016, 2017; Hajba and Guttman, 2016).

Although most reports in the literature mention the usefulness of the microfluidic systems for process screening in enzymatic reactors, very few actually proceed to increase the scale in order to benchmark the results obtained (Bajić et al., 2017). Some previous reports exist on the upscale of chemical reactions (Kockmann, 2016; Jensen, 2017) as well as some fermentation processes (Brás et al., 2016; Hemmerich et al., 2018), however there are very few reports on the upscale from microfluidics to bench-top scales or higher for enzymatic reactions (Prasnikar et al., 2017; Hirama et al., 2017; Novak et al., 2016). This lack of demonstrations of successful upscaling of microfluidic systems for such reactions is part of the motivation of the presented work, where we aim to demonstrate the upscale of packed-bed systems for enzymatic synthesis.

In order to correctly upscale a system, there are certain physical characteristics that should be maintained such as the aspect ratio of the system and flow and mass transport behavior. In order to maintain mass/heat transport consistent in both systems, we first need to characterize the microfluidic system by calculating the Reynolds number $(R e)$ and the Péclet number $(P e)$. In the case of the $P e$, two variants can be calculated, one that is dependent on the particles that make up the packed bed $\left(P e_{P}\right)$, the other relates to the axial dispersion throughout the length of the reactor $\left(P e_{L}\right)$. The method of calculating these dimensionless numbers is presented below (Rastegar and Gu, 2017).

$\operatorname{Re}_{\text {Packed Bed }}=\frac{2 R p \times v \times \rho}{\mu(1-\varepsilon)}$

$\frac{1}{P e_{P}}=\frac{0.7 D}{2 R p \times v}+\frac{\varepsilon}{0.18+R e_{\text {Packed Bed }}^{0.59}}$

$P e_{L}=P e_{P} \frac{L}{2 R p}$

Where $R_{p}$ represents the particle radius, $L$ is the packed bed length, $v$ is the fluid velocity, $D$ is the diffusion coefficient of the substrate, $\epsilon$ is the voidage of the packed bed and $\rho$ is the fluid density.

By using working conditions where these numbers are similar, it can be expected to obtain similar results at different process scales.

Dopamine and its precursor levodopa (L-DOPA) are two important signaling molecules in the human organism, playing roles as neurotransmitters and, in the case of dopamine, a major signaling molecule in the brain reward system (Beaulieu-Boire and Lang, 2015; Volkow et al., 2017; Schultz, 2016). An imbalance of these molecules can cause several types of neurological disorders such as Parkinson's disease (Ruonala et al., 2018). L-DOPA is used as a drug in order to increase dopamine production in Parkinson's disease (LeWitt, 2015). Dopamine is not used in the treatment of these types of diseases due to its inability to cross the brain-blood barrier, however it is used in the treatment of low blood pressure and cardiac arrest (Reyes et al., 2016). Both molecules can be produced enzymatically using tyrosine as a substrate and were chosen as a model for this work due to their clinical relevance.

Throughout this work we will show that is possible to produce not only L-DOPA and dopamine individually using a microfluidic system, but that it is possible to perform the cascade reaction necessary to convert tyrosine into dopamine. In this work we will also demonstrate the possibility of upscaling the system and the efficiency of the microfluidic systems as screening tools for bioprocess development.

\section{Materials and methods}

\subsection{Reagents}

Stock solutions of tyrosine ( $2 \mathrm{mM})$, ascorbic acid (1 mM), levodopa (L-DOPA) $(1 \mathrm{mM})$ and dopamine $(1 \mathrm{mM})$ were prepared in phosphate buffered saline (PBS), while a stock solution of sodium hydroxide (1 M) was prepared using Milli-Q water. All these reagents were acquired from Sigma-Aldrich. To produce L-DOPA, tyrosinase from mushroom was purchased from Sigma-Aldrich, and a stock solution of $2 \mathrm{~g} / \mathrm{L}$ was prepared, while the DOPA-decarboxylase used for the dopamine synthesis was purchased from Antibodies-Online and kept at a concentration of $1 \mathrm{mg} / \mathrm{mL}$ in PBS. Substrate solutions were prepared fresh for each experiment from the aforementioned stocks, where oxygen content of the solutions was determined to be $\sim 160.5 \mu \mathrm{M}$ using an oxygen probe (ElectroLab).

\subsection{Bioreactor microfabrication}

The microfluidic reactors used in this work are comprised of twolevel microfluidic chambers, with heights of $100 \mu \mathrm{m}$ and $20 \mu \mathrm{m}$, respectively. These devices are fabricated using standard soft-lithography techniques as described elsewhere (Brás et al., 2019). The first step was to fabricate two different aluminum hard masks, each with the pattern for one of the different height levels. These hard masks are fabricated by depositing $2000 \AA$ of aluminum by magnetron sputtering (Nordiko 7000), on a glass slide (Corning Eagle glass). The aluminum hard mask was patterned by photolithography using a direct-write lithography system (DWL 2.0, Heidelberg systems) of a positive photoresist (PFR7790 G) followed by wet chemical etching (Gravure Aluminum Etchant; Technic, Microchemicals). The mold is made by first spincoating a $20 \mu \mathrm{m}$ thick layer of SU-8 2015 (Microchem) onto a silicon substrate and exposing to UV light through the first hard mask followed by a post exposure bake and development step in propylene glycol methyl ether acetate (PGMEA, Sigma-Aldrich). Next, a $100 \mu \mathrm{m}$ thick layer of SU-8 50 (Microchem) is spin-coated and patterned with the second mask following similar steps as the first layer. Once the second layer has been developed, the full mold undergoes a final hard bake step at $150{ }^{\circ} \mathrm{C}$. The resulting mold is then placed in a $3 \mathrm{~mm}$ thick acrylic mold which is then filled with PDMS (Sylgard 184, Dow Corning), prepared at a 1:10 ratio with its curing agent and degassed. The PDMS is baked at $70{ }^{\circ} \mathrm{C}$ for $90 \mathrm{~min}$. The microfluidic structures are cut out of the frame and sealed against a $250 \mu \mathrm{m}$ thick PDMS membrane following an oxygen plasma surface treatment (Zhou et al., 2010).

\subsection{Enzyme immobilization}

Silica beads, with a diameter distribution between $40 \mu \mathrm{m}$ and $75 \mu \mathrm{m}$ (97728-U Supelco Analytical (Bellefonte, PA)), functionalized in-house with a primary amine by silanization, were used to electrostatically bind both the tyrosinase and the DOPA-decarboxylase. Enzyme stock solutions were left overnight at room temperature with equal parts (v/ v) of a suspension of the functionalized beads. The use of the stock concentrations of both enzymes was to ensure the saturation of the microbeads, maximizing the amount of enzyme per microreactor. The silica beads were functionalized by exposing them to an oxygen plasma and incubating them in a solution of $2 \%(\mathrm{v} / \mathrm{v})$ of (3-aminopropyl)triethoxysilane (Sigma-Aldrich) in HPLC grade acetone, under constant mixing for $3 \mathrm{~h}$. The beads were than washed and stored in MilliQ-water (Hermanson, 2008). Enzyme concentration per bead was determined indirectly by measuring the concentration of enzyme in the supernatant after immobilization using the Bradford method (Bio-Rad), and by weighing the dry mass of beads used, which also revealed the immobilization yield. The retained activity for tyrosinase was also determined by comparing the activity of the enzyme stock to the enzymatic activity of the supernatant as well as the activity of the microbeads, allowing for the determination of the immobilization efficiency. This was done using a colorimetric assay described elsewhere, where the activity unit (U) is defined as a change of 0.001 of the absorbance at $480 \mathrm{~nm}$ per minute (Brás et al., 2019).

The thermal stability of the immobilized and free enzymes was also determined by keeping both samples at $32.5{ }^{\circ} \mathrm{C}$ with periodic sampling for activity assessment. 


\subsection{Product detection}

L-DOPA was detected via its polymerization reaction when exposed to high pH as described in Hormozi-Nezhad's work et al. (2017). When mixed with $\mathrm{NaOH}$, L-DOPA forms fluorescent nanoparticles that can be detected using a fluorescence microscope. For the L-DOPA detection, the sample under analysis (S.U.A.) was mixed with a $1 \mathrm{M}$ solution of $\mathrm{NaOH}$ at a 1:5 ratio (1 being $\mathrm{NaOH})$. The resulting mixture was then injected into a microfluidic column packed with Q-Sepharose beads and the fluorescence shift was measured using UV excitation $\left(\lambda_{\text {excitation }}=\right.$ $365 \mathrm{~nm}, \lambda_{\text {emission }}=420 \mathrm{~nm}$ ). The rate of fluorescence increase of pure L-DOPA solutions was monitored and used as a calibration curve for determining the reactor output.

\subsection{Production experiments - microscale}

The microfluidic bioreactors were packed with enzyme-functionalized beads by inserting a pipette tip containing the bead suspension into the appropriate inlet of the structure and applying a negative pressure at the outlet with a syringe pump (NE-4000, New Era Pumps). The microfluidic system was maintained at $32.5{ }^{\circ} \mathrm{C}$ throughout the experiments, while the reaction substrate was injected at the appropriate flow rate using a syringe pump. The output of the microreactor was collected at intervals of at least $10 \mathrm{~min}$ in order to provide enough volume for both product detection and enzyme content analysis. The output collected during the first $10 \mathrm{~min}$ interval in all experiments was disregarded as this contains mostly the liquid medium used to pack the beads.

\subsection{Production experiments - milliliter scale}

The milliliter scale version of the packed-bed reactor was a stainlesssteel pre-column typically used for HPLC with an inner diameter of 1 $\mathrm{cm}$ and a total length of $2.5 \mathrm{~cm}$. The column was connected to an AKTA (P-900, GE Healthcare) pumping system to provide adequate pumping at the desired flow rate. Both the bioreactor and the reservoir for the reaction substrate were heated at $32.5{ }^{\circ} \mathrm{C}$ by immersion in a hot bath (VWR). Beads were packed manually into the columns and samples were collected at regular intervals for quantification of product output and free enzyme.

\subsection{Image analysis}

The fluorescence micrographs were analyzed using the open access software ImageJ (Schneider et al., 2012) to quantify the L-DOPA. These images were split into their 3 color channels, RGB, with only the Green channel being used for quantification.

\section{Results and discussion}

\subsection{Reaction pathway}

The use of tyrosinase as a catalyst to produce L-DOPA and as an initial step towards the production of dopamine poses a challenge. On the one hand, the enzyme is cheap enough to be a viable industrial catalyst (Min et al., 2019), but on the other, the lack of specificity towards phenolic compounds other than tyrosine limits its own efficiency as can be seen in Fig. 1.

Tyrosinase participates in several oxidation reactions up until the production of melanin, hence there is a need to add a reducing agent to the reaction medium to avoid further oxidation of L-DOPA (Min et al., 2015). Ascorbic acid was chosen as the reducing agent as it does not produce a toxic by-product and does not appear to interfere with the enzymatic activity of the DOPA-decarboxylase needed for the second step of the reaction.

The second step of the reaction is the decarboxylation of L-DOPA into dopamine using DOPA-decarboxylase. Working conditions for these enzymes ( $\mathrm{pH}$ and temperature) are very similar, thus there is no need for complex buffer exchanges or heating strategies to keep both reactions functioning in sequence (Bertoldi, 2014).

\subsection{L-DOPA and dopamine production-DOPA and dopamine production}

Throughout this section we will explain the method for product detection and the optimizations of the individual reaction steps for both L-DOPA and dopamine before delving into the sequential reaction necessary to produce dopamine from L-tyrosine.

\subsubsection{Detection protocol}

The optimization of the production of both L-DOPA and dopamine consists of retrieving the output of the microfluidic production reactor, which is called the sample under analysis (S.U.A) and detecting the LDOPA content using a smaller microfluidic structure as shown in Fig. 2A.

By mixing the S.U.A with $\mathrm{NaOH}, \mathrm{L}-\mathrm{DOPA}$ and dopamine participate in polymerization reactions as described by Hormozi-Nezhad et al. (2017). By capturing the resulting fluorescent nanoparticles, using a smaller microfluidic column packed with Q-Sepharose microbeads, and monitoring the fluorescence increase over time it is possible to monitor the yield of the microreactor (Fig. 2B). As seen in the supplementary information, the direct detection of dopamine using this method is hindered by the presence of L-DOPA, thus the consumption of L-DOPA was used for the indirect detection of the quantity of dopamine produced. Using a pure solution of L-DOPA, a calibration curve was determined (Fig. 2C). The detection method was tested with different amounts of ascorbic acid present in the solution in order to check for possible interferences, as it is a possible contaminant during the production experiments (Fig. 2C). More extensive results concerning the detection protocol can be found in Section 1 of the Supplementary Information.

\subsubsection{Enzyme immobilization and stability}

The enzyme immobilization was always performed on the day before the start of the experiment. Given the immobilization protocol, the enzyme will adsorb to the surface of the microbeads as well as to the pore walls.

Immobilization of total protein was measured using the Bradford method, producing a yield of $53 \%(\mathrm{~m} / \mathrm{m})$ in the case of tyrosinase and $100 \%(\mathrm{~m} / \mathrm{m})$ in the case of DOPA-decarboxylase. Immobilization yield based on activity measurements resulted in an immobilization yield of $\sim 69 \%(\mathrm{U} / \mathrm{U})$ for tyrosinase and $100 \%$ (U/U) for DOPA-decarboxylase. Efficiency of the immobilization (observed activity/immobilized activity) was determined to be $170 \%$ for tyrosinase and $33 \%$ for DOPAdecarboxylase. Although uncommon, it is possible to obtain efficiencies of immobilization above $100 \%$, this typically being attributed to the support stabilizing the active center of the enzyme or preventing aggregation which could impede the enzyme activity in solution (Mateo et al., 2007; Rodrigues et al., 2013).

Results concerning the thermal stability of tyrosinase can be found in the Supplementary Information, in the case of tyrosinase, no activity loss was seen in the initial $24 \mathrm{~h}$, however there was a drop in activity of $35 \%$ in the time frame between 24 and $36 \mathrm{~h}$ and an additional drop in activity of $37.5 \%$ between 36 and $48 \mathrm{~h}$. In the case of DOPA-decarboxylase this was not attempted as there is no loss of activity to be registered within the time frame of the microfluidic experiments.

\subsubsection{Screening of L-DOPA process conditions}

For the optimization of the reaction conditions, the main parameters of interest include temperature, $\mathrm{pH}$, flow rate, residence time and substrate concentration. For tyrosinase and DOPA-decarboxylase activity, reported optimal values of temperature is approximately $30{ }^{\circ} \mathrm{C}$ and pH between 6 and 8 (Yildiz et al., 2013). With this in mind, 


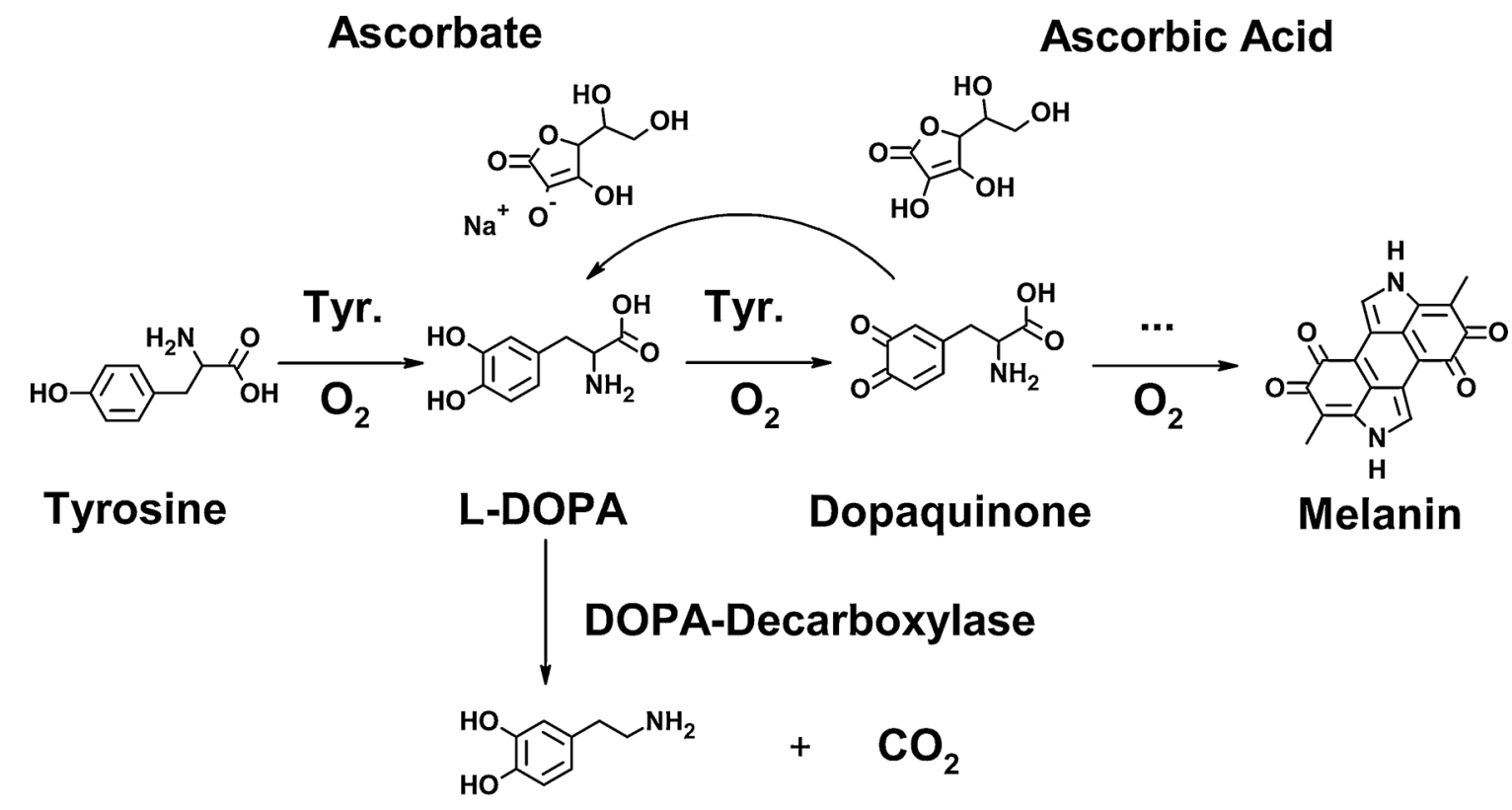

\section{Dopamine}

Fig. 1. L-DOPA and dopamine reaction pathway using tyrosine as a substrate. Tyrosinase plays a part in several of the reactions that take place, which eventually culminate with the production of melanin. Ascorbic acid is added to the reaction as the reducing agent of choice. DOPA-decarboxylase is than used to convert the produced L-DOPA into dopamine, with the release of $\mathrm{CO}_{2}$.

A
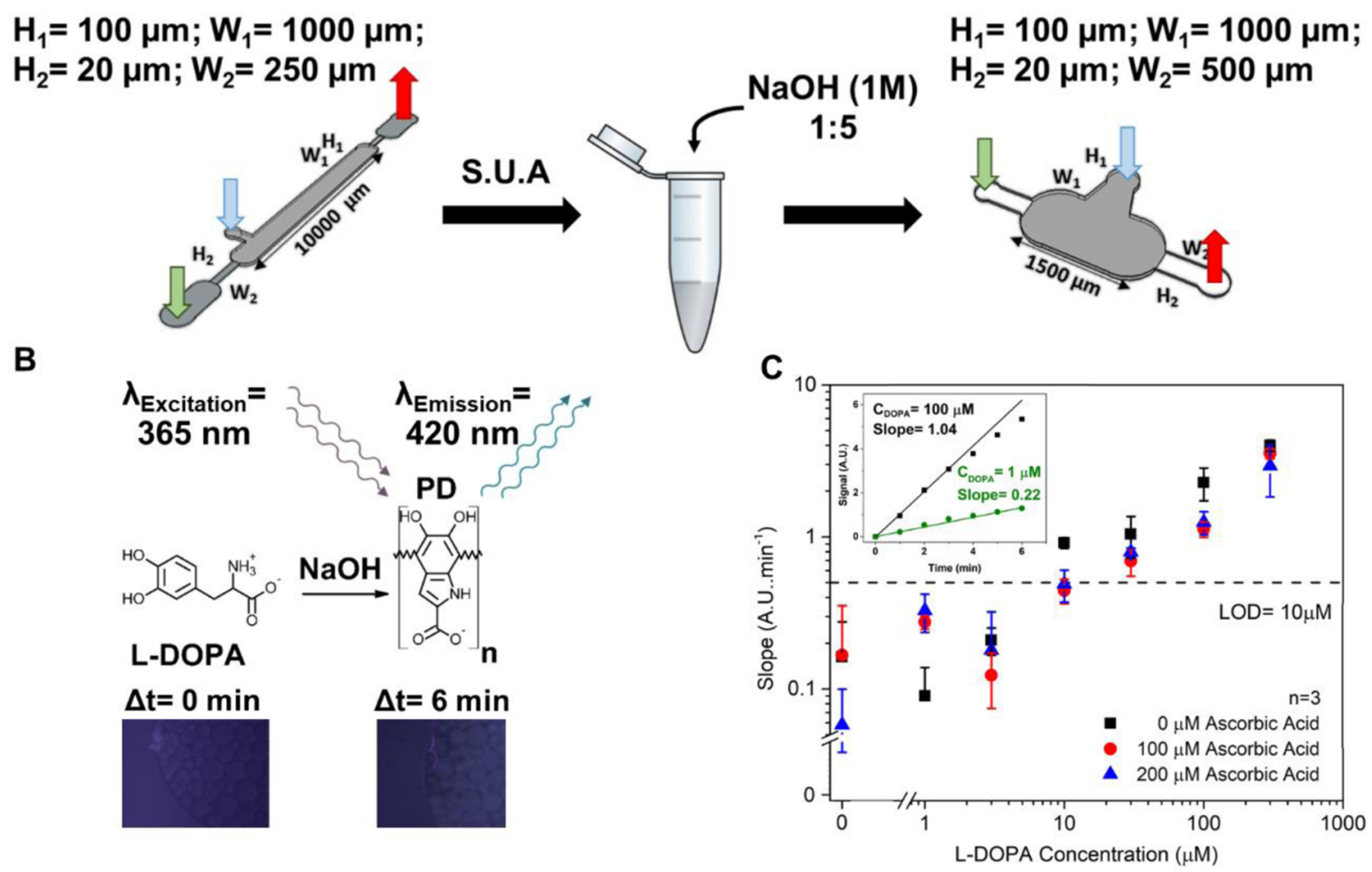

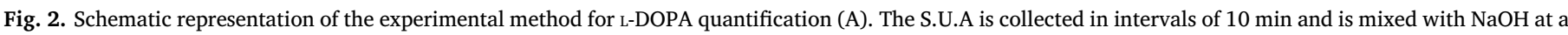

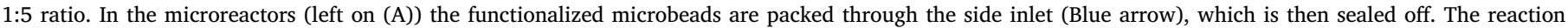

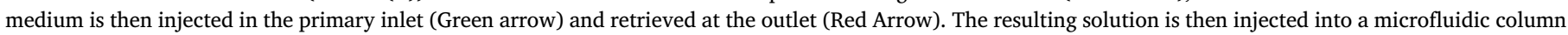

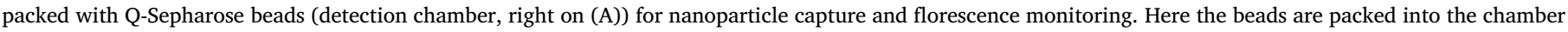

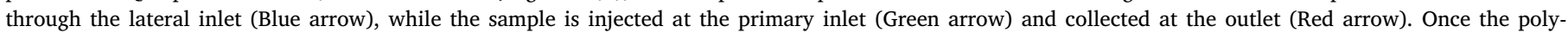

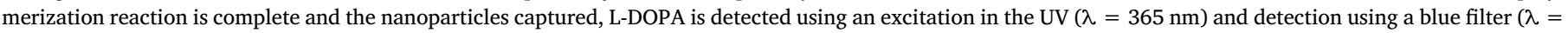

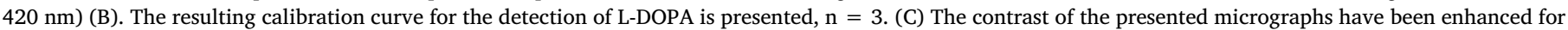
visualization purposes (For interpretation of the references to colour in this figure legend, the reader is referred to the web version of this article). 

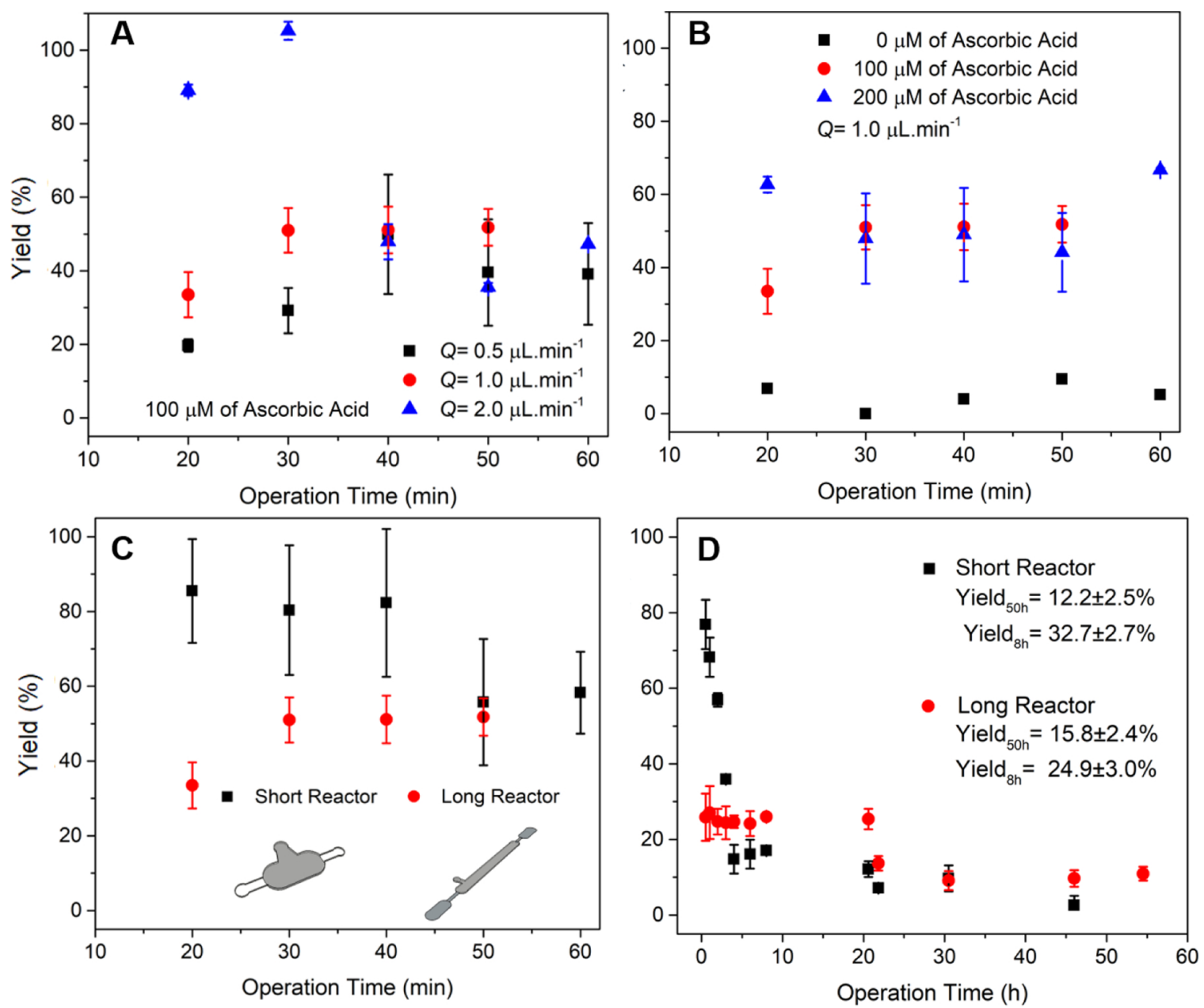

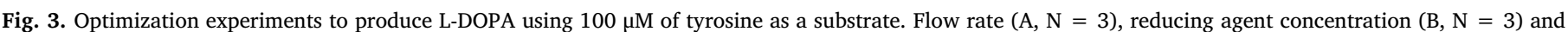

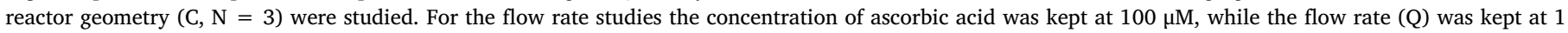

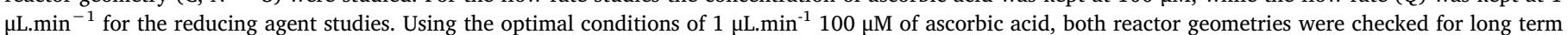
viability (D, $\mathrm{N}=4)$.

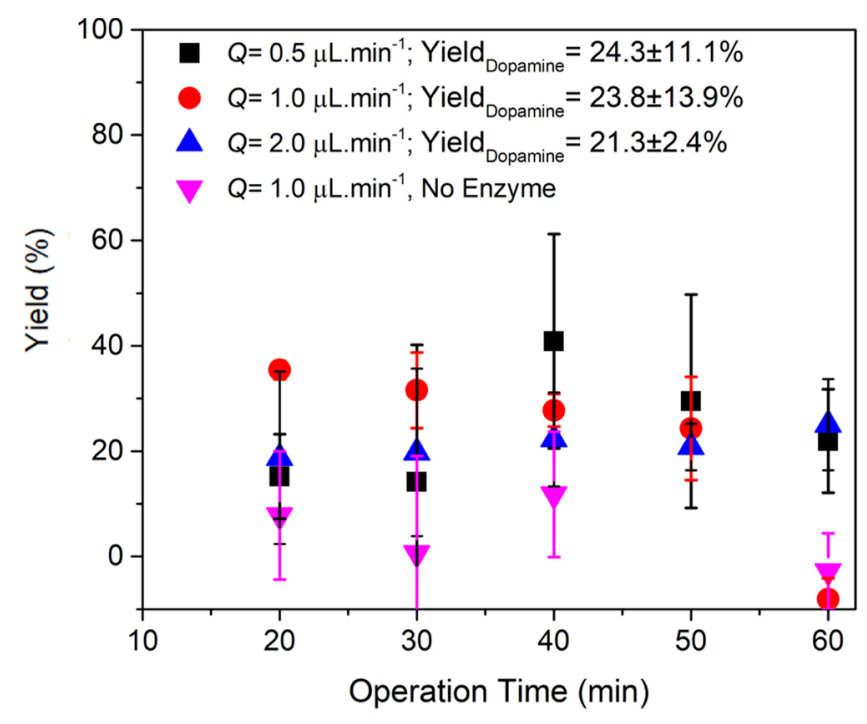

Fig. 4. Flow rate $(Q)$ screening of the dopamine production using $\mathrm{SiO}_{2} \mathrm{mi}-$ crobeads functionalized with DOPA-decarboxylase. A pure L-DOPA solution was used as a substrate and its consumption was monitored using the described method. temperature and $\mathrm{pH}$ were maintained at $32.5^{\circ} \mathrm{C}$ and 7 , respectively. For the production of L-DOPA, oxygen is necessary as an oxidant, the oxygen content was determined to be $160.5 \mu \mathrm{M}$, which prevents reaction limitation on this end. Optimization experiments focused on the variables flow rate, substrate ratio in the reaction medium and reactor geometry. The metric used for the optimization of the microreactor was the yield (\%) defined as the concentration of L-DOPA/dopamine divided by the concentration of the respective substrate being injected into the system. The results are summarized in Fig. 3.

For these optimization experiments, two reactor geometries were considered, the "Long Reactor", which has $L_{\text {Bead Chamber }}=1 \mathrm{~cm}, H=100$ $\mu \mathrm{m}$ and $W=1 \mathrm{~mm}$, while the "Short Reactor" differs only by having a $L=1.5 \mathrm{~mm}$, obtaining volumes of $978.5 \mathrm{~nL}$ and $128.5 \mathrm{~nL}$ respectively. The average voidage of the reactors was determined to be 0.45 , resulting in a dry weight of the microbeads of $187 \mu \mathrm{g}$ and $1426 \mu \mathrm{g}$ for the Short and the Long reactors, respectively. The amount of tyrosinase per bead was determined to be $6.03 \mu \mathrm{g}_{\text {Tyrosinase }} / \mathrm{mg}_{\text {Bead, }}$, resulting in a volumetric activity of $0.186 \mathrm{U} / \mu \mathrm{L}$ and an amount of tyrosinase of $1.1 \mu \mathrm{g}$ in the Short Reactor and $8.6 \mu \mathrm{g}$ in the Long Reactor.

Fig. 3A shows that the working flow rate has a significant impact on the initial moments of the reaction, but after $\sim 30 \mathrm{~min}$ the yield reaches a steady state which is independent of the flow rate. This was not expected, as the lower flow rates increase the residence time inside the reactor, which should increase the probability of the reaction reaching a post-L-DOPA stage due to the catecholase activity of the enzyme (Min 

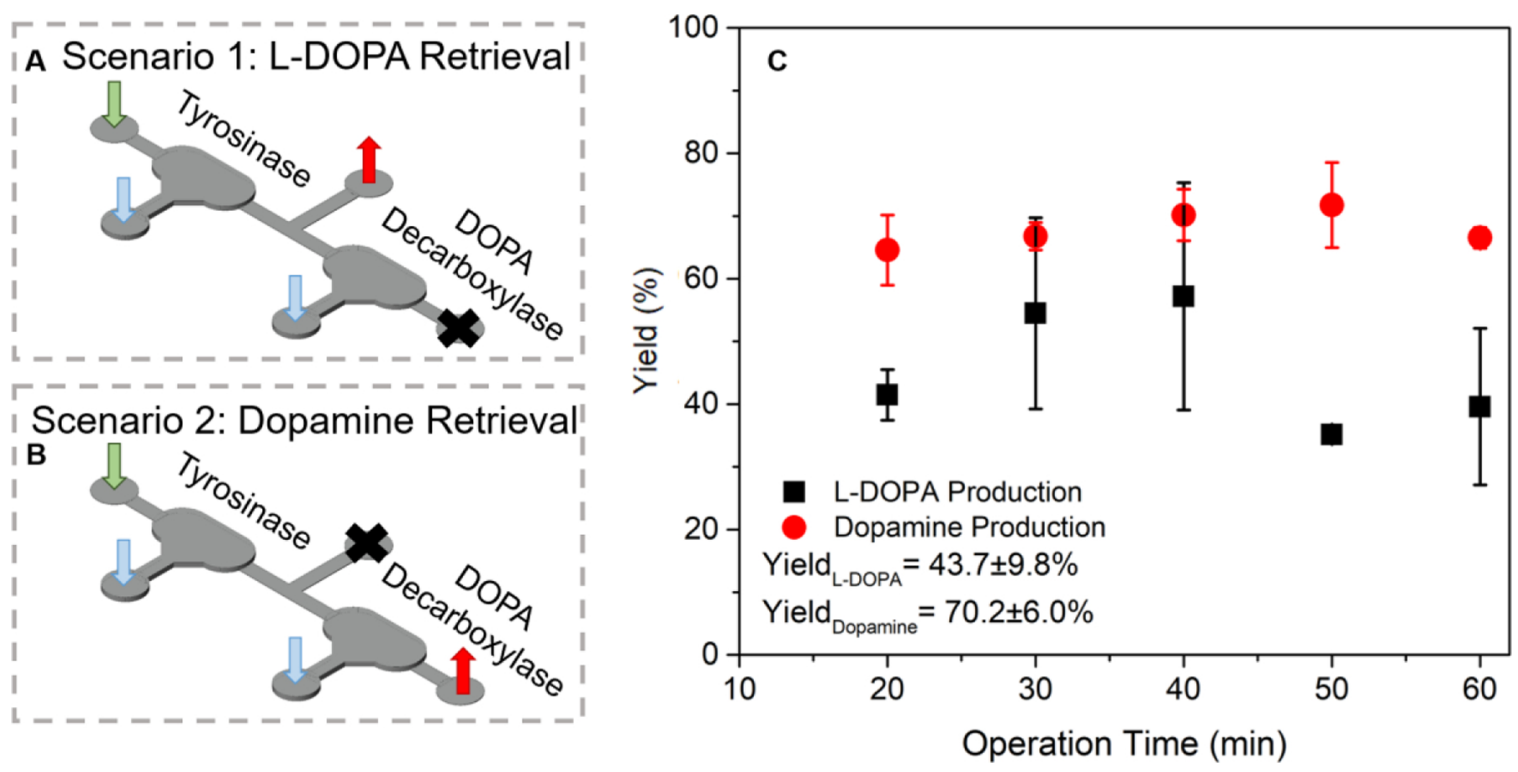

Fig. 5. Sequential production of both L-DOPA and dopamine in a single integrated microfluidic system. The reaction medium is injected at $1 \mu$ L.min ${ }^{-1}$ and is composed of $100 \mu \mathrm{M}$ of tyrosine and $100 \mu \mathrm{M}$ of ascorbic acid. For the monitoring of L-DOPA production, the outlet is sealed off and the middle outlet is used for sample retrieval (A). For the detection of dopamine, the middle outlet is sealed off and the sample is collected at the outlet (B). Productivity for both reaction steps (C). $\mathrm{N}=2$.

Table 1

Flow properties and resulting working flow rates for both micro and milliliter scale.

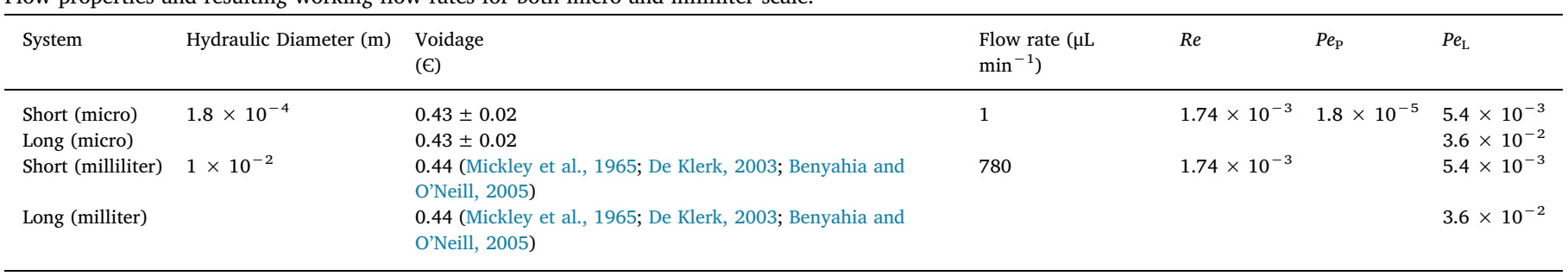

et al., 2015). However, it is possible that the changes in residence time are not sufficient to cause a significant difference in the output of the reactor, or that enough of the reaction by-products have accumulated on the bead surface that introduce some mass transfer limitations to the system. Based on these results, a flow rate of $1 \mu \mathrm{L}$. $\mathrm{min}^{-1}$ was chosen for the subsequent experiments. The impact of the reducing agent, ascorbic acid, in the medium was studied next (Fig. 3B). Fig. 3B shows that in the absence of ascorbic acid, there is nearly no production of L-DOPA. However, increasing the amount of ascorbic acid from $100 \mu \mathrm{M}$ to 200 $\mu \mathrm{M}$ did not further improve the yield. Intrigued by the lack of impact of the residence time on the yield of the microreactor, which was attributed to there being multiple chances of the substrate encountering the enzyme throughout the reactor length, we proceeded to test a shorter microreactor. The yield of the shorter microreactor was compared to that of the longer version, and the results are shown in Fig. 3C. It is possible to observe that the Short Reactor largely outperforms the Long Reactor. This is attributed to the low residence time inside the Short Reactor and to the fact that each substrate molecule will encounter less enzymes in the Short Reactor compared to its longer counterpart, decreasing the possibility of side reactions and further product degradation. In both cases, product degradation may be minimized if the oxygen content of the solution was decreased, in order to avoid further oxidization of the product. The operational stability over longer periods of time is presented in Fig. 3D. It can be seen in both types of microreactors that there is an initial loss of enzymatic activity in the system which wasn't registered in previous experiments which had a shorter time frame. As mentioned before, the thermal stability was tested by keeping the immobilized and also free tyrosinase incubated at $32.5{ }^{\circ} \mathrm{C}$ for $48 \mathrm{~h}$, with no loss of activity registered in the initial $24 \mathrm{~h}$, however with drops of activity after 36 and $48 \mathrm{~h}$. This loss in activity is most likely the reason for the drop in activity registered after the initial $24 \mathrm{~h}$ for both reactors, however does not explain the loss registered in the initial $24 \mathrm{~h}$ time frame. This initial loss of activity can be due to multiple reasons such as fouling of the reactor, preventing newer substrate to reach the enzyme, as can be seen in Section 2 of the Supplementary Information; the suicide nature of tyrosine as a substrate for tyrosinase activity (Muñoz-Muñoz et al., 2010; Vilanova et al., 1984); or enzyme wash-out of the system due to poor interaction between the beads and the enzyme. This last scenario was tested using the Bradford method, and no enzyme was detected in the reactor outflow, as shown in Section 3 of the Supplementary Information, however there could had been washout at concentrations not detectable using the Bradford assay.

\subsubsection{Screening of dopamine process conditions}

To facilitate the integration of the dopamine production step with the L-DOPA step, similar conditions to the ones used for L-DOPA production, namely the flow rate and reactor operation time, were tested. For these experiments, pure L-DOPA was used as a substrate and the results are presented in Fig. 4.

Due to the inability of direct detection of dopamine at this scale, the yield of the conversion of L-DOPA to dopamine was indirectly determined through the consumption of L-DOPA in the system. With that in mind, a control assay without DOPA-decarboxylase present was performed in order to consider any L-DOPA loss due to adsorption to the beads or spontaneous oxidation, which was determined to be between 5 and $10 \%$ of the initial amount of L-DOPA, as seen in Fig. 4 in 

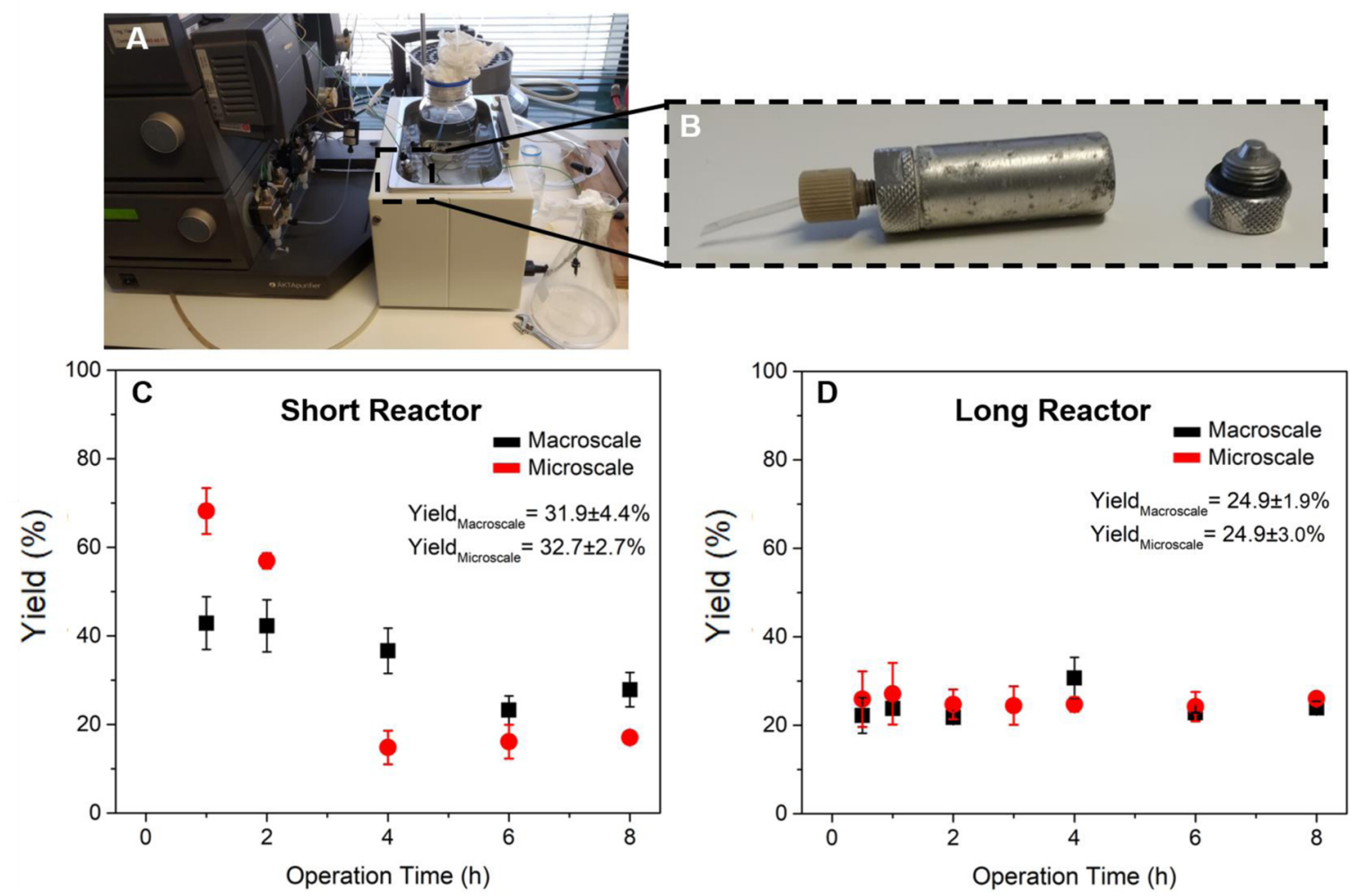

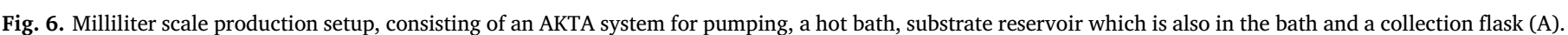

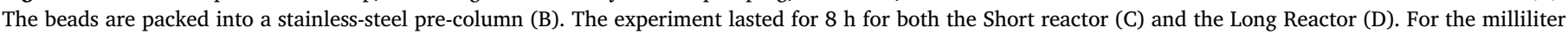
scale experiments, $\mathrm{N}=2$.

Table 2

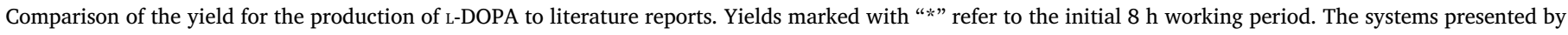
other authors are all macroscale systems.

\begin{tabular}{|c|c|c|c|c|c|}
\hline System & $\begin{array}{l}\text { Yield } \\
(\%)\end{array}$ & $\begin{array}{l}\text { Space-time Yield } \\
\left(\mathrm{mg}_{\mathrm{L}-\mathrm{DOPA}} \mathrm{L}^{-1} \mathrm{~h}^{-1}\right)\end{array}$ & $\begin{array}{l}\text { Biocatalyst Yield } \\
\left(\mathrm{mg}_{\mathrm{L}-\mathrm{DOPA}} \mathrm{mg}^{-1}{ }_{\mathrm{Tyr}} \mathrm{h}^{-1}\right)\end{array}$ & Total L-DOPA produced (mg) & Mode of operation and Geometry \\
\hline Microscale (Short) & $12.2\left(32.7^{*}\right)$ & $4.46 \times 10^{-2}\left(0.806^{*}\right)$ & $131.2\left(351.7^{*}\right)$ & $7.8 \times 10^{-3}\left(3.10 \times 10^{-3 *}\right)$ & Packed Bed, Continuous - 54 h (8 h) \\
\hline Milliliter scale (Short) & 31.9 & 0.786 & 340.9 & 2.36 & Packed Bed, Continuous - 8 h \\
\hline Microscale (Long) & $15.7(24.9 *)$ & $5.73 \times 10^{-2}\left(0.606^{*}\right)$ & $21.6(33.9 *)$ & $1.0 \times 10^{-2}\left(2.33 \times 10^{-3 *}\right)$ & Packed Bed, Continuous - 54 h $(8 \mathrm{~h})$ \\
\hline Milliliter scale (Long) & 24.9 & 0.614 & 34.0 & 1.84 & Packed Bed, Continuous - $8 \mathrm{~h}$ \\
\hline Xu et al. (2012) & 53.0 & 209 & 1045 & 104.5 & CSTR, Batch - 2h \\
\hline Xu et al. (2012) & 28.5 & 103 & 515 & 3.30 & CSTR, Continuous - $8 \mathrm{~h}$ \\
\hline Xu et al. (2012) & 13.5 & 48.9 & 0.88 & 7.60 & Packed Bed, Continuous - 36h \\
\hline Ho et al. (2003) & 44.1 & 5.54 & 0.55 & 1.99 & CSTR, Batch - 36h \\
\hline
\end{tabular}

the "no Enzyme" condition.

Overall the productivity of this system shows an average yield of $\sim 22 \%$. Like in the case of L-DOPA production in this range, the flow rate doesn't have a significant impact on the system output. A flow rate of $1 \mu \mathrm{L} \cdot \mathrm{min}^{-1}$ was chosen for subsequent experiments.

\subsection{Cascade production}

Once the optimization of the production of L-DOPA and dopamine were performed (Section 3.2), the cascade reaction was tested. The system comprising two sequential chambers is schematically shown in Fig. 5A. The first chamber is packed with tyrosinase-functionalized beads, and the second chamber is packed with DOPA-decarboxylasefunctionalized beads. A small outlet between the two chambers allows retrieving samples for L-DOPA production assessment. A total of 4 systems were used simultaneously for these experiments, where samples from the first column were retrieved from 2 of them, which had the second chamber empty and sealed off, for L-DOPA assessment (Fig. 5A), while the remaining structures, had this outlet sealed and were used for dopamine determination at the outlet (Fig. 5B).

Fig. $5 \mathrm{C}$ shows that the dopamine yield is $70 \%$ in relation to the amount of L-DOPA that is produced by the first stage, which is approximately $45 \mu \mathrm{M}$. This productivity is much higher than what was registered when pure L-DOPA at $100 \mu \mathrm{M}$ was used (Fig. 4). This leads the authors to believe that in the case of the experiments depicted in Fig. 4, the DOPA-decarboxylase chamber is being injected with higher concentrations of L-DOPA than it can convert. One hypothesis to improve the yield would be to increase the protein content per bead by improving the immobilization protocol. The overall yield of the combined system is approximately $30 \%$.

The results demonstrate the feasibility of microfluidic packed-bed reactors, not only for single step reactions but also for multistep reaction sequences. One could argue for the use of these types of microreactors for large scale production using complex scaling-out methods. However, the authors believe that the best current use of these microreactors is for the screening of process conditions/enzymes. However, even for screening applications, it is necessary to perform an adequate upscale of the system. This topic is further discussed in the next section. 


\subsection{Upscale production}

As a proof of concept, only the synthesis of L-DOPA was up scaled. Using Eq. (1) through (3) the properties of the microfluidic system were calculated and the necessary working conditions for the bench-scale system were determined. The working conditions are summarized in Table 1.

As can be seen, it is possible to upscale packed-bed reactors while still maintaining the same flow characteristics as the microfluidic system in terms of $P e$ and $R e$ numbers due to the fact that the flow behavior will be dominated by the bead packing instead of the reactor geometry, as would be the case for plug flow reactors and tank reactors.

The milliliter scale version of the bioreactor consists of a chromatography pre-column packed with the functionalized beads. In order to prevent the washout of the beads through the reactor outlet, a small piece of cotton was placed at the bottom of the bed, the height of the bed was fixed by using an appropriate volume of beads. Production experiments were carried out for $8 \mathrm{~h}$ and compared to their microscale counterparts, as shown in Fig. 6.

The yield of the Short reactor (Fig. 5C) shows a good correlation at both scales, with similar losses of enzymatic activity being registered. This activity loss is probably due to the accumulation of reaction byproducts as mentioned previously. As for the Long version of the reactor (Fig. 5D), the results obtained at the microscale match the results obtained at the larger scale for the entire duration of the experiment. By looking at these results, it is safe to say that by using a microfluidic system and maintaining similar mass transport conditions, it is possible to accurately predict process outcome at the milliliter scale for packedbed reactors.

The microreactor, as well as the milliliter reactor, were then compared in Table 2 to other enzymatic reactors reported in the literature, using the yield, as well as the space-time yield and biocatalyst yield as figures of merit. While the system presented in this work has similar yield as other reports in the literature, it has inferior performance when the space-time yield is accounted for, especially when compared to a batch system. However, the system presented in this work shows comparable output or even largely outperforms other continuous systems in terms of biocatalyst yield. It can also be seen in Table 2 that the space-time yield and biocatalyst yield are very similar between the microscale and milliliter approach within this work, further validating the microfluidic system as a useful screening tool for enzymatic reactions.

\section{Conclusions}

Throughout this work a microfluidic approach to the optimization of biochemical enzymatic production was demonstrated. We successfully demonstrated the use of microfluidic bioreactors for 2 single step reactions, the generation of L-DOPA using tyrosine as a substrate and the production of dopamine using L-DOPA. In addition, feasibility of using this type of system for a multi-step production scheme was demonstrated by converting tyrosine into dopamine in a continuous mode in a two-chamber microfluidic reactor. In all these reactions, the yields obtained were like those obtained by other macroscale works reported in the literature. This work also demonstrated the successful upscale of the system, obtaining similar results at both the microscale and at milliliter scale to produce L-DOPA.

\section{Declaration of Competing Interest}

The authors declare that they have no known competing financial interests or personal relationships that could have appeared to influence the work reported in this paper.

\section{Acknowledgements}

The authors wish to thank Filipe Carvalho for his help in troubleshooting during the upscale process. The authors also wish to thank the Fundação para a Ciência e a Técnologia (FCT) for funding through a $\mathrm{PhD}$ grant for E.J.S. Bras within the AIM Doctoral Program (PD/BD/ 128167/2016), the research projects OptLoC (PTDC/BBB-NAN/5927/ 2014) and the Research Units (UID/NAN/50024 and UID/Multi/ 04046/2013). Funding for the project POINT4PAC (SAICTPAC/0019/ 2015 and LISBOA-01-0145-FEDER-016405) was through European Structural \& Investment Funds through the COMPETE Programme and from National Funds through FCT - Fundação para a Ciência $e$ a Tecnologia.

\section{Appendix A. Supplementary data}

Supplementary material related to this article can be found, in the online version, at doi:https://doi.org/10.1016/j.jbiotec.2020.07.016.

\section{References}

Asanomi, Y., Yamaguchi, H., Miyazaki, M., Maeda, H., 2011. Enzyme-Immobilized Microfluidic Process Reactors. pp. 6041-6059. https://doi.org/10.3390/ molecules16076041.

Bajić, M., Plazl, I., Stloukal, R., Žnidaršič-Plazl, P., 2017. Development of a miniaturized packed bed reactor with $\omega$-transaminase immobilized in LentiKats ${ }^{\circledast}$. Process Biochem. 52 (January), 63-72. https://doi.org/10.1016/j.procbio.2016.09.021.

Beaulieu-Boire, I., Lang, A.E., 2015. Behavioral effects of levodopa. Mov. Disord. 30 (January (1)), 90-102. https://doi.org/10.1002/mds.26121.

Benyahia, F., O’Neill, K.E., 2005. Enhanced voidage correlations for packed beds of various particle shapes and sizes. Part. Sci. Technol. 23 (2), 169-177.

Bertoldi, M., 2014. Mammalian dopa decarboxylase: structure, catalytic activity and inhibition. Arch. Biochem. Biophys. 546 (March), 1-7. https://doi.org/10.1016/J.ABB. 2013.12.020.

Bolivar, J.M., Tribulato, M.A., Petrasek, Z., Nidetzky, B., 2016. Let the substrate flow, not the enzyme: practical immobilization of $\mathrm{d}$-amino acid oxidase in a glass microreactor for effective biocatalytic conversions. Biotechnol. Bioeng. 113 (November (11)), 2342-2349. https://doi.org/10.1002/bit.26011.

Bolivar, J.M., Luley-Goedl, C., Leitner, E., Sawangwan, T., Nidetzky, B., 2017. Production of glucosyl glycerol by immobilized sucrose phosphorylase: options for enzyme fixation on a solid support and application in microscale flow format. J. Biotechnol. 257 (September), 131-138. https://doi.org/10.1016/J.JBIOTEC.2017.01.019.

Brás, E.J., Chu, V., Aires-Barros, M.R., Conde, J.P., Fernandes, P., 2016. A microfluidic platform for physical entrapment of yeast cells with continuous production of invertase. J. Chem. Technol. Biotechnol. https://doi.org/10.1002/jctb.5010.

Brás, E.J.S., Fortes, A.M., Chu, V., Fernandes, P., Conde, J.P., 2019. Microfluidic device for the point of need detection of a pathogen infection biomarker in grapes. Analyst. https://doi.org/10.1039/C9AN01002E.

Choi, J.-M., Han, S.-S., Kim, H.-S., 2015. Industrial applications of enzyme biocatalysis: current status and future aspects. Biotechnol. Adv. 33 (November (7)), 1443-1454. https://doi.org/10.1016/J.BIOTECHADV.2015.02.014.

De Klerk, A., 2003. Voidage variation in packed beds at small column to particle diameter ratio. AIChE J. 49 (8), 2022-2029.

den Haan, R., van Rensburg, E., Rose, S.H., Görgens, J.F., van Zyl, W.H., 2015. Progress and challenges in the engineering of non-cellulolytic microorganisms for consolidated bioprocessing. Curr. Opin. Biotechnol. 33 (June), 32-38. https://doi.org/10.1016/J. COPBIO.2014.10.003.

Du, L., et al., 2018. Microfluidic reactor for lipase-catalyzed regioselective synthesis of neohesperidin ester derivatives and their antimicrobial activity research. Carbohydr. Res. 455 (January), 32-38. https://doi.org/10.1016/J.CARRES.2017.11.008.

Fuchs, M., Farnberger, J.E., Kroutil, W., 2015. The industrial age of biocatalytic transamination. Eur. J. Org. Chem. 2015 (November (32)), 6965-6982. https://doi.org/10. 1002/ejoc. 201500852.

Giovannini, P.P., et al., 2019. Continuous production of eugenol esters using enzymatic packed-bed microreactors and an evaluation of the products as antifungal agents. Flavour Fragr. J. 34 (May (3)), 201-210. https://doi.org/10.1002/ffj.3492.

Gruber, P., et al., 2017. Conscious Coupling: the Challenges and Opportunities of Cascading Enzymatic Microreactors. no. May. https://doi.org/10.1002/biot. 201700030.

Gruber, P., et al., 2018. Enzymatic synthesis of chiral amino-alcohols by coupling transketolase and transaminase-catalyzed reactions in a cascading continuous-flow microreactor system. Biotechnol. Bioeng. 115 (March (3)), 586-596. https://doi.org/ 10.1002/bit.26470.

Hajba, L., Guttman, A., 2016. Continuous-flow biochemical reactors: biocatalysis, bioconversion, and bioanalytical applications utilizing immobilized microfluidic enzyme reactors. J. Flow Chem. 6 (March (1)), 8-12. https://doi.org/10.1556/1846.2015. 00028.

Hemmerich, J., Noack, S., Wiechert, W., Oldiges, M., 2018. Microbioreactor systems for accelerated bioprocess development. Biotechnol. J. 13 (April (4)), 1700141. https:// 
doi.org/10.1002/biot.201700141.

Hermanson, G.T., 2008. Bioconjugate Techniques, 2nd ed. Pierce Biotechnology.

Hirama, H., et al., 2017. Design, fabrication, and performance of an optimized flow reactor with parallel micropacked beds. Ind. Eng. Chem. Res. 56 (48), 14200-14206.

Ho, P.Y., Chiou, M.S., Chao, A.C., 2003. Production of L-DOPA by tyrosinase immobilized on modified polystyrene. Appl. Biochem. Biotechnol. 111 (3), 139-152. https://doi. org/10.1385/ABAB:111:3:139.

Hormozi-Nezhad, M.R., Moslehipour, A., Bigdeli, A., 2017. Simple and rapid detection of 1-dopa based on in situ formation of polylevodopa nanoparticles. Sens. Actuators B Chem. 243 (May), 715-720. https://doi.org/10.1016/J.SNB.2016.12.059.

Jensen, K.F., 2017. Flow chemistry-microreaction technology comes of age. AIChE J. 63 (3), 858-869.

Kockmann, N., 2016. Modular equipment for chemical process development and smallscale production in multipurpose plants. ChemBioEng Rev. 3 (February (1)), 5-15. https://doi.org/10.1002/cben.201500025.

LeWitt, P.A., 2015. Levodopa therapy for Parkinson's disease: pharmacokinetics and pharmacodynamics. Mov. Disord. 30 (January (1)), 64-72. https://doi.org/10.1002/ mds. 26082 .

Liu, S., 2012. Bioprocess Engineering: Kinetics, Biosystems, Sustainability, and Reactor Design. Newnes.

Luckarift, H.R., Ku, B.S., Dordick, J.S., Spain, J.C., 2007. Silica-immobilized enzymes for multi-step synthesis in microfluidic devices. Biotechnol. Bioeng. 98 (October (3)), 701-705. https://doi.org/10.1002/bit.21447.

Madarász, J., Németh, D., Bakos, J., Gubicza, L., Bakonyi, P., 2015. Solvent-free enzymatic process for biolubricant production in continuous microfluidic reactor. J. Clean. Prod. 93 (April), 140-144. https://doi.org/10.1016/J.JCLEPRO.2015.01.028.

Mateo, C., Palomo, J.M., Fernandez-Lorente, G., Guisan, J.M., Fernandez-Lafuente, R., 2007. Improvement of enzyme activity, stability and selectivity via immobilization techniques. Enzyme Microb. Technol. 40 (May (6)), 1451-1463. https://doi.org/10. 1016/j.enzmictec. 2007.01.018.

Mickley, H.S., Smith, K.A., Korchak, E.I., 1965. Fluid flow in packed beds. Chem. Eng. Sci. 20 (3), 237-246. https://doi.org/10.1016/0009-2509(65)80034-3.

Min, K., Park, K., Park, D.-H., Yoo, Y.J., 2015. Overview on the biotechnological production of l-DOPA. Appl. Microbiol. Biotechnol. 99 (January (2)), 575-584. https:// doi.org/10.1007/s00253-014-6215-4.

Min, K., Park, G.W., Yoo, Y.J., Lee, J.-S., 2019. A perspective on the biotechnological applications of the versatile tyrosinase. Bioresour. Technol. 289 (October), 121730 https://doi.org/10.1016/J.BIORTECH.2019.121730.

Muñoz-Muñoz, J.L., et al., 2010. Suicide inactivation of the diphenolase and monophenolase activities of tyrosinase. IUBMB Life 62 (June (7)), 539-547. https://doi. org/10.1002/iub.348.

Novak, U., Lavric, D., Žnidaršiè-Plazl, P., 2016. Continuous lipase B-catalyzed isoamyl acetate synthesis in a two-liquid phase system using Corning ${ }^{\oplus} \mathrm{AFR}^{\mathrm{TM}}$ module coupled with a membrane separator enabling biocatalyst recycle. J. Flow Chem. 6 (1), 33-38.

Prasnikar, A., Urbic, T., Plazl, I., 2017. Microscale technology and biocatalytic processes (Part $2 *$ ): scale-up case of surface enzyme-catalyzed biotransformation. Chim. OGGIChem. Today 35 (4), 12-14.

Rastegar, S.O., Gu, T., 2017. Empirical correlations for axial dispersion coefficient and
Peclet number in fixed-bed columns. J. Chromatogr. A 1490 (March), 133-137. https://doi.org/10.1016/J.CHROMA.2017.02.026.

Reyes, A.J., Ramcharan, K., Harnarayan, P., Mooteeram, J., 2016. Symmetrical digital gangrene after a high dose intravenous infusion of epinephrine and dopamine following resuscitation from cardiac arrest. BMJ Case Rep. 2016 (November). https:/ doi.org/10.1136/bcr-2016-217977. p. bcr2016217977.

Rodrigues, R.C., Ortiz, C., Berenguer-Murcia, Á., Torres, R., Fernández-Lafuente, R., 2013. Modifying enzyme activity and selectivity by immobilization. Chem. Soc. Rev. 42 (August (15)), 6290-6307. https://doi.org/10.1039/c2cs35231a.

Ruonala, V., Pekkonen, E., Airaksinen, O., Kankaanpää, M., Karjalainen, P.A., Rissanen, S.M., 2018. Levodopa-induced changes in electromyographic patterns in patients with advanced Parkinson's disease. Front. Neurol. 9 (February), 35. https://doi.org/ 10.3389/fneur.2018.00035.

Schneider, C.A., Rasband, W.S., Eliceiri, K.W., 2012. NIH Image to ImageJ: 25 years of image analysis. Nat. Methods 9 (July (7)), 671-675. https://doi.org/10.1038/nmeth. 2089.

Schultz, W., 2016. Dopamine reward prediction-error signalling: a two-component response. Nat. Rev. Neurosci. 17 (March (3)), 183-195. https://doi.org/10.1038/nrn. 2015.26.

Strniša, F., Bajić, M., Panjan, P., Plazl, I., Sesay, A.M., Žnidaršič-Plazl, P., 2018. Characterization of an enzymatic packed-bed microreactor: experiments and modeling. Chem. Eng. J. 350, 541-550.

Sun, H., Zhang, H., Ang, E.L., Zhao, H., 2018. Biocatalysis for the synthesis of pharmaceuticals and pharmaceutical intermediates. Bioorg. Med. Chem. 26 (April (7)), 1275-1284. https://doi.org/10.1016/J.BMC.2017.06.043.

Vilanova, E., Manjon, A., Iborra, J.L., 1984. Tyrosine hydroxylase activity of immobilized tyrosinase on enzacryl-AA and CPG-AA supports: stabilization and properties. Biotechnol. Bioeng. 26 (November (11)), 1306-1312. https://doi.org/10.1002/bit. 260261107.

Volkow, N.D., Wise, R.A., Baler, R., 2017. The dopamine motive system: implications for drug and food addiction. Nat. Rev. Neurosci. 18 (December (12)), 741-752. https:// doi.org/10.1038/nrn.2017.130.

Wang, J., et al., 2019. A biocatalytic hydroxylation-enabled unified approach to C19hydroxylated steroids. Nat. Commun. 10 (December (1)), 3378. https://doi.org/10. 1038/s41467-019-11344-0.

Xu, D.-Y., Chen, J.-Y., Yang, Z., 2012. Use of cross-linked tyrosinase aggregates as catalyst for synthesis of 1-DOPA. Biochem. Eng. J. 63 (April), 88-94. https://doi.org/10. 1016/J.BEJ.2011.11.009.

Yildiz, H.B., Caliskan, S., Kamaci, M., Caliskan, A., Yilmaz, H., 2013. 1-Dopa synthesis catalyzed by tyrosinase immobilized in poly(ethyleneoxide) conducting polymers. Int. J. Biol. Macromol. 56 (May), 34-40. https://doi.org/10.1016/J.IJBIOMAC.2013. 01.031 .

Zhou, J., Ellis, A.V., Voelcker, N.H., 2010. Recent developments in PDMS surface modification for microfluidic devices. Electrophoresis 31 (January (1)), 2-16. https://doi. org/10.1002/elps.200900475.

Zhu, C., et al., 2019. Enzyme immobilized on the surface geometry pattern of groove-typed microchannel reactor enhances continuous flow catalysis. J. Chem. Technol Biotechnol. 94 (May (8)). https://doi.org/10.1002/jctb.6053. p. jctb.6053. 